\title{
Zyklodestruktive Verfahren zur Senkung des Augeninnendrucks - eine Übersicht
}

\section{Cyclodestructive Procedures to Lower Intraocular Pressure - A Review}

\author{
Autoren \\ Drazen Jurjevic, Jens Funk, Marc Töteberg-Harms \\ Institut \\ Augenklinik, UniversitätsSpital Zürich, Schweiz \\ Schlüsselwörter \\ Glaukom, Kryokoagulation, Mikropuls, Photokoagulation, \\ Zyklodestruktion
}

Key words

glaucoma, cryocoagulation, micropulse, photocoagulation, cyclodestruction

$\begin{array}{ll}\text { eingereicht } & 12.12 .2016 \\ \text { akzeptiert } & 23.1 .2017\end{array}$

Bibliografie

DOI http://dx.doi.org/10.1055/s-0043-105271

Online-publiziert 9.6.2017 | Klin Monatsbl Augenheilkd 2019; 236: 63-68 @ Georg Thieme Verlag KG Stuttgart · New York । ISSN 0023-2165

\section{Korrespondenzadresse}

Dr. med. Marc Töteberg-Harms, FEBO

Augenklinik, UniversitätsSpital Zürich

Frauenklinikstrasse 24, 8091 Zürich, Schweiz

Tel.: + 41/(0)44/2551111, Fax: + 41/(0) 44/2554472

marc.toeteberg@usz.ch

\section{ZUSAMMENFASSUNG}

Die einzige Therapie des Glaukoms, für die es eine ausreichende Evidenz gibt, ist die Senkung des Augendrucks. Die Augendrucksenkung kann durch eine Verbesserung des Abflusses oder eine Verringerung der Kammerwasserproduktion erzielt werden. Chirurgisch kann eine Verringerung der Kammerwasserproduktion durch zyklodestruktive Verfahren erreicht werden. Dieser Artikel soll eine kurze Zusammenfassung über die verschiedenen zyklodestruktiven Verfahren geben. Die Effektivität der Zyklophotokoagulation ist hinreichend belegt.
Durch Anpassung der Laserprotokolle hat sich das Risikoprofil reduzieren lassen. Die in frühen Studien beschriebenen gravierenden Komplikationen bis hin zu Visusverlust sind selten geworden. Das Indikationsspektrum der Zyklophotokoagulation hat sich von End-Stage-Glaukomen bis zu Glaukomen mit gutem Visus erweitert. Selbst der Einsatz zyklodestruktiver Verfahren bei pädiatrischen Glaukomen hat an Akzeptanz gewonnen. Eine vielversprechende nächste Generation mikrogepulster Diodenlaser könnte bei gleicher Wirksamkeit das Risikoprofil noch weiter reduzieren. Die Nichtunterlegenheit der Mikropulszyklophotokoagulation gegenüber den etablierten Verfahren wurde in ersten Studien unter Beweis gestellt. Zum gegenwärtigen Zeitpunkt fehlen noch Langzeitstudien bezüglich Effektivität und Nebenwirkungsprofil sowie ein standardisiertes Laserprotokoll.

\section{ABSTRACT}

The only therapy for glaucoma, for which there is sufficient evidence, is the lowering of intraocular pressure. Intraocular pressure reduction can be achieved by an improvement in outflow or a reduction of aqueous humor production. Surgically, a reduction of aqueous humor production can be achieved by cyclodestructive procedures. This article provides a brief summary of the different cyclodestructive procedures. The efficacy of cyclophotocoagulation is well documented. The risk profile has been reduced by changes in the laser protocols. Serious complications described in early studies (e.g., visual loss) rarely occur nowadays. The indication spectrum of cyclophotocoagulation has expanded from end-stage glaucoma to glaucoma with good visual acuity. Even the use of cyclodestructive procedures in pediatric glaucoma has gained acceptance. A promising next generation of micropulsed diode lasers could further reduce the risk profile with the same effectiveness. The non-inferiority of micropulse cyclophotocoagulation versus the established procedures has been proven in early studies. However, studies with long-term follow-up and on side-effects are still lacking. Furthermore, a standardized laser protocol must be established. 


\section{Einführung}

Nur die Senkung des Augendrucks in der Therapie des Glaukoms ist mit Evidenz belegt worden [1-3]. Der Augendruck ist durch ein Gleichgewicht zwischen Kammerwasserbildung und -abfluss determiniert [4]. Im Rahmen der Glaukomtherapie gibt es generell 2 mögliche Ansätze, den Augeninnendruck zu senken: 1.) Eine Steigerung des Abflusses des Kammerwassers oder 2.) eine Verringerung der Produktion des Kammerwassers. Die Verringerung der Produktion des Kammerwassers im Ziliarkörper wird chirurgisch durch zyklodestruktive Verfahren erreicht. Dieser Artikel soll eine kurze Zusammenfassung über die verschiedenen zyklodestruktiven Verfahren und ihre Unterschiede geben.

\section{Transsklerale Zyklophotokoagulation mittels „G Probe Glaucoma Device“}

Bei der transskleralen Zyklophotokoagulation (ts-CPC) wird ein Diodenlaser $(810 \mathrm{~nm})$ zur Verödung des Ziliarkörpers eigesetzt. Eine kleine Fiberoptik nach Gaasterland (G-Probe, > Abb. 1) wird dabei auf den Bulbus und die Konjunktiva aufgesetzt. Dies garantiert einen Abstand von ca. 1,2 mm zum chirurgischen Limbus [5, 6]. Die Fußplatte der Sonde, die in der Kurvatur des Bulbus geformt ist, komprimiert hierbei die konjunktivalen und epi-/skleralen Blutgefäße, sodass die pigmentierten Hämatozyten nicht die Laserenergie absorbieren. Hierdurch durchdringt der Laserstrahl sämtliche Augenschichten und trifft auf pigmentierte Zellen im Ziliarkörperepithel, welche die Energie schlussendlich absorbieren. Es kommt zur Hitzeentwicklung. Das Ziel ist die fokale Koagulation des Ziliarkörpers mit konsekutiver geringerer Kammerwasserproduktion und Augendrucksenkung. Liu et al. fanden einen erhöhten Vorderkammer-Flare in den ersten Tagen, der mit der Zeit reversibel war und binnen ca. 1 Monats wieder verschwand. Die gleiche Gruppe fand Nekrose im Koagulationsbereich mit Ausnahme der Sklera unmittelbar nach der Behandlung. Circa 1 Woche nach CPC fanden sich im behandelten Gebiet Makrophagen. Nach 3-5 Wochen verschwanden die koagulierten Ziliarkörperzotten, die nach 3-6 Monaten durch eine flache Narbe ersetzt wurden [7].

Die Lokalisation des Ziliarkörpers kann mittels Transillumination erfolgen, bei der ein dunkler Ring dessen Lage anzeigt [8]. Insbesondere bei atypischer Bulbusmorphologie (bei hoher Myopie oder Hyperopie, kongenitalem Glaukom usw.) sollte man sich nicht auf den vorgegebenen Abstand vom Limbus verlassen, sondern die Transillumination zur Lokalisation des Ziliarkörpers zur Hilfe nehmen. Eine Weiterentwicklung der G-Probe-Sonde mit eingebauter Transilluminationsmöglichkeit wäre wünschenswert. Hyperpigmentierte Bindehautveränderungen oder subkonjunktivale Blutungen sollten ausgespart werden, um lokale Verbrennungen an diesen Orten zu vermeiden [8]. Der Laser wird i. d. R. anfänglich auf $2000 \mathrm{~mW}$ und $2500 \mathrm{msec}$ eingestellt. Sollte es zur Gasbildung durch zu hohe Energie mit hörbarem „Pop-Effekt“ kommen, so ist die Energie nach 2 konsekutiven Pop-Effekten in 100-mW-Schritten zu reduzieren, bis der Pop-Effekt bei den restlichen Herden ausbleibt. Des Weiteren wurde kein signifikanter Unterschied im drucksenkenden Effekt im Rahmen der Be-

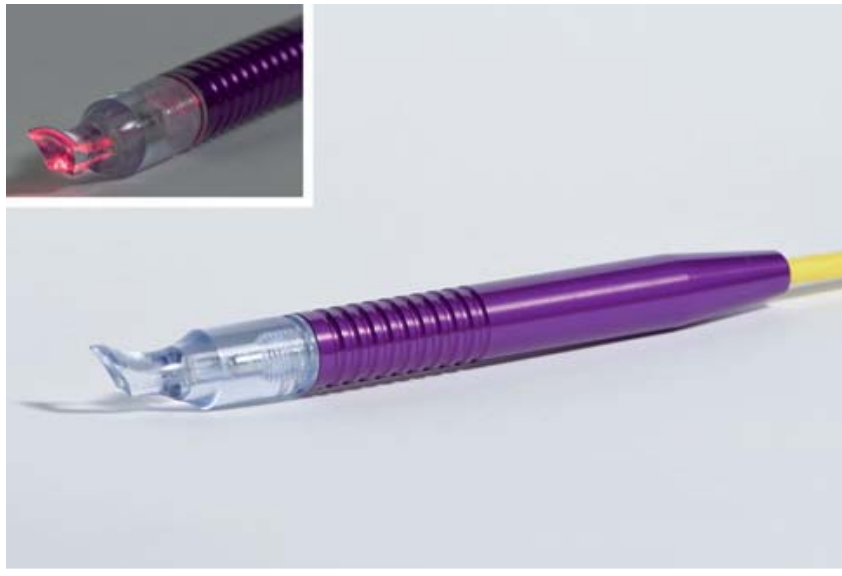

- Abb. 1 G-Probe-Handstück (Iridex) mit gekrümmter Fußplatte, die auf den Bulbus am Limbus aufgesetzt wird. Hierdurch wird der Laser ca. 1,2 mm ab Limbus appliziert [6].

handlung mit oder ohne Pop-Effekt festgestellt [9]. In einer Behandlung werden meist zwischen 10 und 20 Herde über $120^{\circ}$ bis maximal $270^{\circ}$ der Limbuszirkumferenz appliziert. Die Laserapplikation bei 3 und 9 Uhr sollte vermieden werden, um die langen Ziliarnerven zu schonen und eine vermehrte Inflammation und Pupillenschädigung postoperativ zu vermeiden. Auch die superioren 2 bis 3 Uhrzeiten sollten mindestens für eine spätere Trabekulektomie ausgespart werden. Manche Chirurgen sparen aus diesem Grunde auch die komplette superiore Hemizirkumferenz aus, da i. d. R. nach CPC ausgeprägte Vernarbungen von Konjunktiva, Tenon und Sklera resultieren. Eine potenzielle Auswirkung auf nachfolgende mikrochirurgische Glaukomprozeduren im Trabekelmaschenwerk und Suprachoroidalraum ist denkbar, Daten hierzu gibt es bisher allerdings nicht. Nicht selten sind mehrere Sitzungen an einem Auge möglich und nötig, um die angestrebte Drucksenkung zu erreichen [10]. Die Wirksamkeit einer 2. CPC ist nach Erfahrung der Autoren schlechter als die primäre CPC. Daten für adulte Glaukome sind uns nicht bekannt. Der gleiche Effekt wurde auch für kongenitale Glaukome in der Literatur beschrieben [11].

Da es sich bei der ts-CPC um ein schmerzhaftes Verfahren handelt, ist im Vorfeld eine ausreichende Anästhesie und Analgesie erforderlich, wobei i.d.R. eine para- oder retrobulbäre Anästhesie der subkonjunktivalen Anästhesie aufgrund der besseren Analgesie vorzuziehen ist. Es bedarf i.d.R. keiner Allgemeinanästhesie. Auch auf eine ausreichende postoperative Analgesie mit nicht steroidalen Analgetika ist zu achten.

In den letzten Jahren konnten zahlreiche durchgeführte Studien die Effektivität der ts-CPC unter Beweis stellen. Im Rahmen von prospektiven Untersuchungen wurden drucksenkenden Effekte mit einer Erfolgsrate von bis zu $50 \%$ über 2 Jahre beschrieben [12]. Nach knapp mehr als 2 Sitzungen konnten Augendrucksenkungen von bis zu 50\% über 84 Monate erreicht werden [13]. Gleiche Erfolgsraten bei jedoch kürzerem Follow-up (ca. 67 Monate) zeigte die Behandlung des kongenitalen Glaukoms [11]. Kontroverse Ergebnisse von erfolgreicher bis unbefriedigender IOPSenkung (IOP: intraocular pressure, Augendruck) fanden sich im Rahmen der Behandlung uveitischer Glaukome mit der ts-CPC 


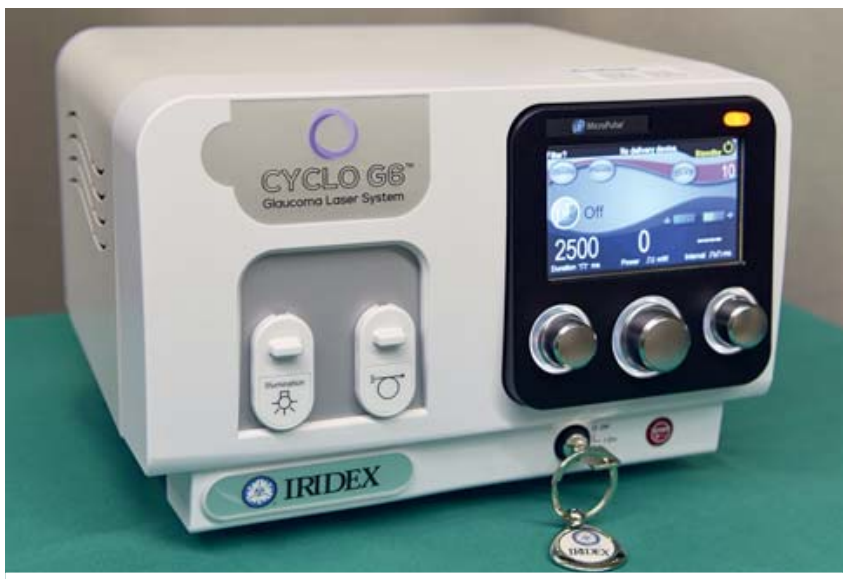

- Abb. 2 Cyclo G6 Glaucoma Laser System der Firma Iridex für die Zyklophotokoagulation. Das Gerät kann sowohl für die transskerale als auch für die Mikropulszyklophotokoagulation verwendet werden. Neben einem Ausgang für Laserenergie gibt es zusätzlich einen Kaltlichtleiter, der prinzipiell z. B. für die Applikation mit Diaphanoskopie genutzt werden kann.

$[14,15]$. Der Vergleich von Effektivität und Komplikationsrate der ts-CPC gegenüber dem Ahmed-Valve-Implantat (AVI) bei fortgeschrittenem Glaukom zeigte eine ähnliche Langzeitdrucksenkung (ts-CPC: 61,18\% und AVI: 59,26\%) [16].

Heute kommen zyklodestruktive Verfahren, insbesondere die ts-CPC, aufgrund ihrer Verfügbarkeit und Effektivität in vielen Situationen zum Einsatz. So z. B.:

- therapierefraktäre (auch pädiatrische Glaukome) oder fortgeschrittene Glaukome mit schlechter Visusprognose

- therapierefraktäre Glaukome nach Versagen von fistulierenden Operationen oder von Shunt-Implantaten

- Glaukome, bei denen ein fistulierender Eingriff aufgrund von Nebenerkrankungen, Bindehaunarben oder auf Patientenwunsch nicht möglich ist oder abgelehnt wird

- Neovaskularisationsglaukom (NVG)

- posttraumatische Glaukome

- Glaukome nach perforierender Keratoplastik

- Sekundärglaukome bei Silikonölfüllung

- uveitische Sekundärglaukome

- Notfallsituationen

- Tropfenunverträglichkeit, wenn ein fistulierender Eingriff nicht möglich ist

In den letzten Jahren ist man immer mehr davon abgekommen, die ts-CPC nur als End-Stage-Option in der Behandlung des Claukoms zu betrachten. Untersuchungen im Rahmen der Behandlung früher Glaukome mit gutem präoperativem Visus zeigten positive Ergebnisse in Bezug auf die Drucksenkung mit vertretbarer Komplikationsrate [17]. Die Evaluation der ts-CPC als Ersteingriff im Rahmen therapierter Glaukome zeigte bei Ansari et al. eine IOP-Reduktion von $43 \%$ nach durchschnittlich 12 Monaten mit vertretbarer Komplikationsrate von $9 \%$, Hypotonien traten dabei nicht auf [18]. In 58\% der Fälle konnten antihypertensive Tropfen eingespart und in allen Fällen orales Acetazolamid gestoppt wer-

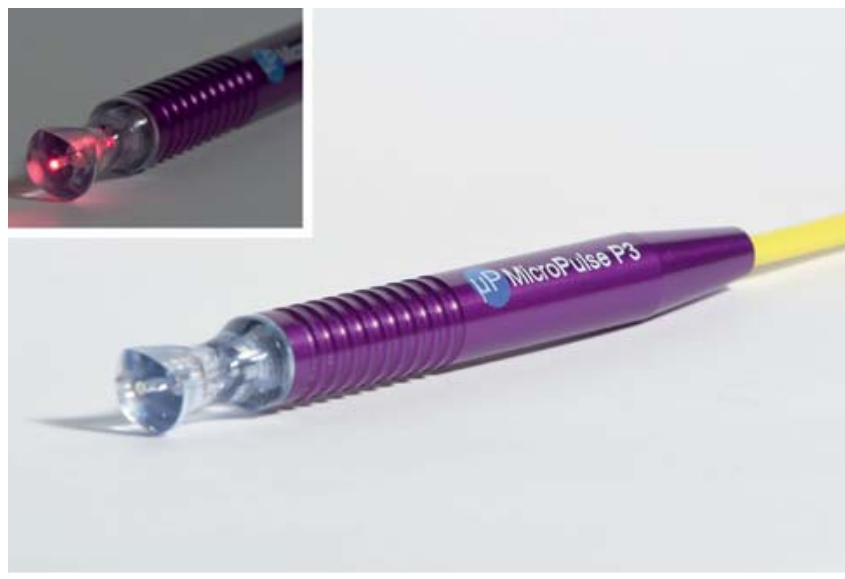

- Abb. 3 MP3-Handstück (Iridex) für die mikrogepulste transsklerale Zyklophotokoagulation.

den. Des Weiteren zeigte sich eine Visusminderung in 13\% der behandelten Patienten, die als Folge von Kataraktentwicklung oder Glaukomprogression gewertet wurde [18, 19].

\section{Mikrogepulste transsklerale Zyklophotokoagulation}

Bei der mikrogepulsten transskleralen Zyklophotokoagulation (mpts-CPC) wird die Laserenergie nicht kontinuierlich, sondern gepulst alternierend mit Pausen appliziert (on-/off-cycle). Zur Verwendung kommt, wie bei der konventionellen ts-CPC, ein Diodenlaser im Infrarotspektrum $(810 \mathrm{~nm})$. Somit wird die Energie weiterhin von melaninhaltigen Zellen absorbiert. In der Theorie sollen die zyklischen „on-/off“-Phasen genau so viel Energie im Zielgewebe aufbauen, dass es zur Ablation des Ziliarkörperepithels kommt und die Produktion des Kammerwassers reduziert wird. Das umliegende Gewebe, welches durch den niedrigeren Melaningehalt weniger Energie absorbiert, wird dadurch geschont, dass der "off“-Zyklus bereits eingetreten ist, bevor die Temperatur zu hoch wird [20,21]. Durch Schonung des perifokalen Gewebes, des Ziliarkörperstromas, der Muskeln usw. ist der postoperative Entzündungsreiz deutlich verringert. Dies sollte zu einem reduzierten Schmerzempfinden führen [20].

Für den Einsatz der Mikropulstechnologie wird das kommerziell erhältliche Lasersystem „Cyclo G6 Glaucoma Laser System“ der Firma Iridex verwendet $(\bullet$ Abb. 2 ). Im Vorfeld der mpts-CPC sollte eine Analgosedierung oder eine Lokalanästhesie (parabulbär, retrobulbär oder subkonjunktival) erfolgen. Zu Beginn wird das Handstück ( $\triangleright$ Abb. 3 ) senkrecht und etwa 1-2 mm vom Limbus entfernt auf den Bulbus aufgesetzt. Mit "senkrecht" ist gemeint: senkrecht zum Limbus und senkrecht zur Bulbusoberfläche. Die Energie wird nach dem Standardprotokoll auf $2000 \mathrm{~mW}$ gesetzt. Die Dauer des „on“-Zyklus beträgt etwa $0,5 \mathrm{~ms}$, die des "off“-Zyklus etwa $1,1 \mathrm{~ms}$ (=31,33\% duty cycle). Nacheinander werden die untere und die obere Hemizirkumferenz (unter Aussparung der 3- und 9-Uhr-Position) behandelt. Dazu wird das Handstück unter dauerndem Kontakt und mit leichtem Druck im 
Halbkreis in der oberen/unteren Hemizirkumferenz kontinuierlich hin und her geschwenkt, wobei ein Schwenken in einer Hemizirkumferenz ca. 10 Sekunden dauert. Die Gesamtapplikationsdauer pro Hemizirkumferenz liegt bei ca. 80 Sekunden, es werden aber auch Applikationen von bis zu 120 Sekunden angewandt, z. B. bei Neovaskularisationsglaukom.

Die bisher veröffentlichen Ergebnisse scheinen vielversprechend und mindestens ebenbürtig zur ts-CPC zu sein. Erste Studien belegen eine IOP-Reduktion von etwa $40 \%$ bei einer gleichzeitigen Medikamentenreduktion von ca. 30\% innerhalb der ersten 2 Monate [21]. Andere Arbeiten zeigten eine IOP-Reduktion von 33,3\% innerhalb einer Nachkontrollzeit von 18 Monaten [20]. Tan et al. berichteten über die Schmerzen im Rahmen der Zyklophotokoagulation [20]: Keiner der Patienten hat den postoperativen Schmerz als mäßig oder stark empfunden. Erfreulicherweise wurden keine schwerwiegenden Komplikationen wie Visusverlust oder postoperative Phthisis bulbi beschrieben [20]. Die Indikation ist vergleichbar zur ts-CPC, wobei aufgrund des wahrscheinlich besseren Risikoprofils (weniger Schmerzen und weniger Inflammation) ein Einsatz in früheren Glaukomstadien und nicht nur als Ultima Ratio prinzipiell denkbar wäre.

\section{Endoskopische Zyklophotokoagulation}

Bei der endoskopischen Zyklophotokoagulation (ECP) wird wie bei der ts-CPC ein Diodenlaser zur thermischen Gewebedestruktion verwendet. Die ECP ist ein intraokularer Eingriff. Die Zyklodestruktion erfolgt unter direkter Visualisierung der Ziliarkörperzotten mit einem Endoskop. Prinzipiell gibt es 3 Zugänge: 1.) von der Pars plana aus oder 2.) durch einen Vorderkammerzugang mit limbalem Schnitt oder 3.) Clear-Cornea-Schnitt. Beim anterioren Zugang führt man die ECP-Sonde zwischen der Iris und der Linse an den Ziliarkörper heran, was den Zugang prinzipiell eher für pseudophake oder aphake Auge qualifiziert. Beim Zugang über die Pars plana muss das Auge zumindest anterior vitrektomiert sein. Der wohl größte Vorteil der ECP ist die direkte Visualisierung ( $\vee$ Abb. 4 b) und die dadurch akkuratere Koagulation der Ziliarkörperzotten ( $\vee$ Abb. $\mathbf{4 c}$ ). Die Energie und Bestrahlungsdauer sind dabei so zu wählen, dass man die Weißfärbung und Schrumpfung der Zotten sieht. In einer Sitzung werden i. d. R. 180-270 des Ziliarkörpers koaguliert. Möchte man $360^{\circ}$ koagulieren, sollte man 2 gegenüberliegende Zugänge wählen. In der Regel beginnt man mit 0,2 W Energie und erhöht je nach Ergebnis bis auf ca. 1,2 W. Im Gegensatz zu den nicht visualisierenden transskleralen zyklodestruktiven Verfahren nutzt man die Nähe zum Zielort, um die Laserenergie zu reduzieren und um das umgebende Gewebe zu schonen mit der Absicht, Komplikationen zu reduzieren [22].

Der IOP-reduzierende Effekt der ECP ließ sich in vielen Arbeiten belegen [23]. Retrospektive Studien bei Patienten mit therapierefraktären Glaukomen nach Behandlung mit ECP zeigten eine IOPReduktion von ca. 34\% inklusive einer signifikanten Medikamentenreduktion mit einer Erfolgsrate von 82\% nach 2 Jahren ohne gravierende Komplikationen wie Hypotonie oder Phthisis bulbi [24]. Bei fortgeschrittenen Glaukomen zeigte sich ein vergleichbarer drucksenkender Effekt zwischen Ahmed-Valve-Implantaten und ECP bei niedrigerer Komplikationsrate in der ECP-Gruppe
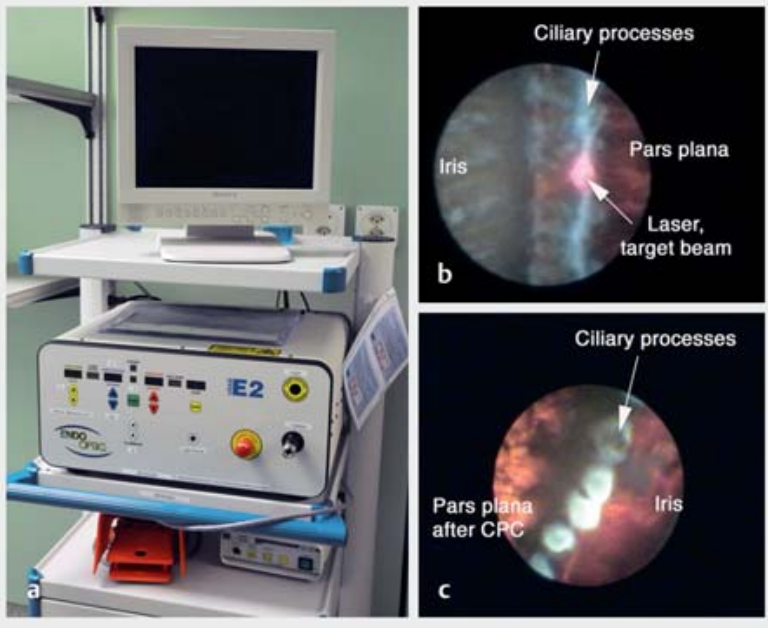

- Abb. 4 Endozyklophotokoagulationseinheit: Turm bestehend aus Laser und Monitor (a). Mittels der endoskopischen Optik werden die Ziliarkörperzotten visualisiert (b) und mittels eines Zielstrahls anvisiert. Die Koagulation erfolgt von der Zottenspitze bis zur Zottenbasis und resultiert in einer Weißfärbung (c).

[25]. In einer prospektiven Studie wurde eine „sanfte“ ECP in Kombination mit einer Phakoemulsifikation gegenüber einer alleinigen Phakoemulsifikation bei kontrollierten Glaukomen verglichen. Francis et al. zeigten einen signifikant niedrigeren IOP und niedrigere Medikamenteneinnahme ohne wesentliche Unterschiede in Visus und Komplikationsrate nach einer Kontrollzeit von 2 Jahren [26].

Einige Autoren beschreiben Vorteile der ECP bei der Behandlung therapierefraktärer pädiatrischer Glaukome. Ein Argument für die ECP ist die gezielte Koagulation des Ziliarkörpers unter Schonung des umliegenden Gewebes. Die erste Arbeit zu diesem Thema aus dem Jahre 1999 untersucht 10 Augen, die mit ECP behandelt wurden [27]. Davon erreichten 5 Augen den gewünschten Zieldruck mit oder ohne Medikation. Bei keinem der behandelten Augen trat eine visusbedrohende Komplikation auf. Bei therapierefraktären pädiatrischen Glaukomen verglichen Kraus et al. den IOP-senkenden Effekt zwischen ts-CPC und ECP. Sie fanden eine Drucksenkung von ca. $29 \%$ bei ts-CPC und $33 \%$ bei ECP ohne einen statistisch signifikanten Unterschied und einem vertretbaren Risikoprofil [28]. Die wohl größten Nachteile der ECP sind der invasive Charakter dieses intraokulären Eingriffs, die Anschaffungs- und Unterhaltskosten und eine flache Lernkurve im Vergleich zu transskleralen Verfahren. Daher wird die ECP bevorzugt nach Versagen von ts-CPC oder fistulierenden Operationen eingesetzt [5].

\section{Zyklokryokoagulation}

Bereits 1950 wurde durch Bietti ein zyklodestruktives Verfahren durch Vereisung des Ziliarkörpers mit Reduktion der Kammerwasserproduktion beschrieben. Gegenüber der damals gängigen Diathermie schien die neue Methode nebenwirkungsärmer. Genau 
wie bei der Zyklophotokoagulation wird ein Handstück dem Bulbus aufgesetzt. Es konnte gezeigt werden, dass die Nebenwirkungen mit der Größe der Kryokoagulationsherde korrelieren [29]. Mit den Jahren hat sich ein Kopfdurchmesser von 2,5 mm mit einem Applikationsabstand von 1,5 bis $4,0 \mathrm{~mm}$ vom Limbus etabliert [30]. Auch im Rahmen der Zyklokryokoagulation empfiehlt es sich, mittels Transillumination den Ziliarkörper zu lokalisieren, um die Effektivität zu steigern und mehrmalige Kryobehandlungen zu vermeiden [29]. Die Kälteapplikationsdauer sollte bis 60 Sekunden betragen, wobei eine längere Dauer mit mehr Nebenwirkungen einhergeht [31]. Während der Kälteapplikation sollte ein gewisser Druck auf die Sklera ausgeübt werden, um die Thermoregulation der lokalen Blutzirkulation zu minimieren [32]. Zur Vereisung des Ziliarkörpers wird eine Temperatur von $-80^{\circ} \mathrm{C}$ empfohlen [29]. Behandelt werden i. d. R. $180^{\circ}$ der Limbuszirkumferenz mit ca. $6 \mathrm{Her}-$ den [30]. Bei ausgedehnteren Behandlungen von bis zu $360^{\circ}$ sind deutlich höhere Komplikationsraten beschrieben. Bei ungenügender IOP-Reduktion lässt sich der Eingriff wiederholen [33].

Um das Maximum der Augendrucksenkung der 1. Sitzung abzuwarten, sollte 4 Wochen bis zur nächsten Behandlung zugewartet werden. Bei gleichem drucksenkendem Effekt zeigt die Zyklokryotherapie jedoch eine höhere Komplikationsrate als die ts-CPC, weshalb die Zyklophotokoagulation als zyklodestruktives Verfahren heute präferiert wird [34,35].

\section{Komplikationen und Nachsorge}

Alle der oben aufgeführten zyklodestruktiven Verfahren können Komplikationen hervorrufen. Durch den lokalen thermischen Effekt sind insbesondere benachbarte Strukturen vulnerabel. Somit ist bei jedem Eingriff mit einer Schädigung der Ziliarkörpermuskeln, des Stromas der Iris, der Bindehaut und der Sklera sowie der angrenzenden Gefäße, Nerven und der Retina zu rechnen. Reaktive Uveitiden, Pupillenverziehungen, Blutungen, chronische Makulaödeme und Schmerzen werden in unterschiedlicher Häufigkeit und Intensität in Abhängigkeit von zugeführter Energie und Ausdehnung der Koagulationsareale beschrieben [5, 24,36]. Pupillenverziehungen und Sphinkterstörungen bis hin zu -lähmungen sind häufig und können zu Blendung und Visusbeeinträchtigung führen [37]. Nach CPC wird häufig eine Visusreduktion gefunden, die nicht immer transient ist [37-39]. Widersprüchlicherweise fand eine Studie mit einer Nachbeobachtungszeit von 2 Jahren am Ende der Nachbeobachtungszeit keine signifikante Visusreduktion [40]. Dennoch sollte das vorhandene Risikoprofil kritisch betrachtet werden, insbesondere, wenn bei jungen Patienten, Patienten mit gutem Visus und Patienten mit noch vorhandener Akkommodationsfähigkeit ein zyklodestruktiver Eingriff evaluiert wird.

Im Rahmen der transskleralen Eingriffe sollten Areale bei 3 und 9 Uhr ausgespart werden, um die langen posterioren Ziliarnerven, Ziliargefäße sowie die sensiblen Trigeminusnerven zur Innervation der Hornhaut zu schonen. Zu bedenken gilt, dass im Rahmen einer ausgedehnten Behandlung oder nach mehrmaligen Operationen der Ziliarkörper so stark geschädigt werden kann, dass es zu einer irreversiblen Hypotonie mit konsekutiver Phthisis bulbi oder sogar zu einer Phthisis dolorosa kommen kann.
Postoperativ ist die Applikation von Steroiden (z. B. Prednisolonacetat $1 \%$ ) und Mydriatika (Atropinsulfat 1\%) für bis zu 4 Wochen zu empfehlen. Einige Autoren empfehlen die einmalige Gabe von Acetazolamid $500 \mathrm{mg}$ postoperativ, um perioperative Tensiospitzen zu vermeiden. Innerhalb der 1. postoperativen Woche sollte mindestens eine Kontrolle stattfinden. In der Regel wird das behandelte Auge bis zum Nachlassen der Wirksamkeit der Anästhesie oder über Nacht mit einem Augenverband abgedeckt. Da postoperativ starke Schmerzen auftreten können, sollte der Patient auch im ambulanten Setting ausreichend mit Analgetika (z. B. NSAID) versorgt sein. Die topischen Steroide sollten je nach Reizzustand langsam ausgeschlichen werden. Da die postoperative Wirkung der zyklodestruktiven Verfahren ihr Maximum gelegentlich erst nach > 4 Wochen erreicht, ist es sinnvoll, die zuvor verordneten Antiglaukomatosa erst im Verlauf langsam zu reduzieren.

\section{Schlussfolgerung}

Durch einfache Handhabung, Flexibilität, niedrige Kosten bei gleichzeitig effizienter IOP-Reduktion und aufgrund der Wiederholbarkeit konnten sich die zyklodestruktiven Verfahren und insbesondere die Zyklophotokoagulation mit dem 810-nm-Diodenlaser als drucksenkender Eingriff etablieren. Durch den Einsatz zyklodestruktiver Verfahren können Augentropfen reduziert und die systemische Einnahme von Acetazolamid sogar gestoppt werden [18]. Dies geht häufig mit einer Verbesserung der Lebensqualität einher. Seit der Einführung des 810-nm-Diodenlasers hat sich das Risikoprofil in zahlreichen Studien mit Anpassung des Laserprotokolls reduzieren lassen. Gefürchtete Komplikationen bis hin zum Visusverlust sind seltener geworden. Wenn sie auftreten, dann am ehesten noch im Rahmen fortgeschrittener Glaukome mit schlechter Visusprognose.

Aufgrund von vertretbaren Komplikationsraten hat sich das Indikationsspektrum zyklodestruktiver Verfahren von ehemals EndStage-Glaukomen hin zu Glaukomen mit noch gutem Visus erweitert. Kraus et al. beschreiben die Wirksamkeit der IOP-Senkung bei gleichzeitig zufriedenstellendem Risikoprofil der Zyklophotokoagulation und schlussfolgern, dass der Einsatz der ts-CPC und ECP sogar als First-Line-Therapie in allen Formen des kindlichen Glaukoms vertretbar sei [28].

Idealerweise soll ein zyklodestruktiver Eingriff bei möglichst niedriger Komplikationsrate den IOP zufriedenstellend reduzieren. Das Ziel der nächsten Generation zyklodestruktiver Systeme ist die weitere Reduktion von Komplikationen durch die gepulste Laserapplikation. Die Nichtunterlegenheit der mpts-CPC gegenüber der etablierten ts-CPC ließ sich in ersten Studien mit kurzem Kontrollintervall belegen. Die Gewebeschonung durch die gepulste Laserapplikation scheint plausibel. Zum gegenwärtigen Zeitpunkt fehlen jedoch Langzeitstudien bez. Effektivität und Nebenwirkungsprofil sowie ein standardisiertes Laserprotokoll.

Interessenkonflikt

MTH erhielt Vortragshonorare und Reisespesen von Iridex in 2016 und 2017. 


\section{Literatur}

[1] Heijl A, Leske MC, Bengtsson B et al. Reduction of intraocular pressure and glaucoma progression: results from the Early Manifest Glaucoma Trial. Arch Ophthalmol 2002; 120: 1268-1279

[2] Epstein DL, Krug JH, Hertzmark E et al. A long-term clinical trial of timolol therapy versus no treatment in the management of glaucoma suspects. Ophthalmology 1989; 96: 1460-1467

[3] Kass MA, Gordon MO, Hoff MR et al. Topical timolol administration reduces the incidence of glaucomatous damage in ocular hypertensive individuals: a randomized, double-masked, long-term clinical trial. Arch Ophthalmol 1989; 107: 1590-1598

[4] Ridley F. The intraocular pressure and drainage of the aqueous humour. Br J Ophthalmol 1930; 11: 217

[5] Pastor SA, Singh K, Lee DA et al. Cyclophotocoagulation: a report by the American Academy of Ophthalmology. Ophthalmology 2001; 108: 2130-2138

[6] Gaasterland DE, Pollack IP. Initial experience with a new method of laser transscleral cyclophotocoagulation for ciliary ablation in severe glaucoma. Trans Am Ophthalmol Soc 1992; 90: 225-243

[7] Liu G], Mizukawa A, Okisaka S. Mechanism of intraocular pressure decrease after contact transscleral continuous-wave Nd:YAG laser cyclophotocoagulation. Ophthalmic Res 1994; 26: 65-79

[8] Sharkey JA, Murray TG. Identification of the ora serrata and ciliary body by transillumination in eyes undergoing transscleral fixation of posterior chamber intraocular lenses. Ophthalmic Surg 1994; 25: 479-480

[9] Rebolleda G, Muñoz F], Murube J. Audible pops during cyclodiode procedures. J Glaucoma 1999; 8: 177-183

[10] Winkler NFK, Funk J. Transsklerale Zyklophotokoagulation als primäre antiglaukomatöse Operation. Klin Monatsbl Augenheilkd 2013; 230: 353-357

[11] Autrata R, Rehurek J. Long-term results of transscleral cyclophotocoagulation in refractory pediatric glaucoma patients. Ophthalmologica 2003; 217: 393-400

[12] Kosoko O, Gaasterland DE, Pollack IP et al. Long-term outcome of initial ciliary ablation with contact diode laser transscleral cyclophotocoagulation for severe glaucoma. Ophthalmology 1996; 103: 1294-1302

[13] Vernon SA, Koppens JM, Menon G] et al. Diode laser cycloablation in adult glaucoma: long-term results of a standard protocol and review of current literature. Clin Exp Ophthalmol 2006; 34: 411-420

[14] Heinz C, Koch J, Heiligenhaus A. Transscleral diode laser cyclophotocoagulation as primary surgical treatment for secondary glaucoma in juvenile idiopathic arthritis: high failure rate after short term follow up. $\mathrm{Br}$ j Ophthalmol 2006; 90: 737-740

[15] Schlote T, Derse M, Zierhut M. Transscleral diode laser cyclophotocoagulation for the treatment of refractory glaucoma secondary to inflammatory eye diseases. Br J Ophthalmol 2000; 84: 999-1003

[16] Yildirim N, Yalvac IS, Sahin A et al. A comparative study between diode laser cyclophotocoagulation and the Ahmed glaucoma valve implant in neovascular glaucoma: a long-term follow-up. J Glaucoma 2009; 18: 192-196

[17] Egbert PR, Fiadoyor S, Budenz DL et al. Diode laser transscleral cyclophotocoagulation as a primary surgical treatment for primary open-angle glaucoma. Arch Ophthalmol 2001; 119: 345-350

[18] Ansari E, Gandhewar J. Long-term efficacy and visual acuity following transscleral diode laser photocoagulation in cases of refractory and non-refractory glaucoma. Eye (Lond) 2007; 21: 936-940

[19] Lai JS, Tham CC, Chan JC et al. Diode laser transscleral cyclophotocoagulation as primary surgical treatment for medically uncontrolled chronic angle closure glaucoma: long-term clinical outcomes. J Glaucoma 2005; 14: 114-119
[20] Tan AM, Chockalingam M, Aquino MC et al. Micropulse transscleral diode laser cyclophotocoagulation in the treatment of refractory glaucoma. Clin Exp Ophthalmol 2010; 38: 266-272

[21] Kuchar S, Moster MR, Reamer CB et al. Treatment outcomes of micropulse transscleral cyclophotocoagulation in advanced glaucoma. Lasers Med Sci 2016; 31: 393-396

[22] Uram M. Ophthalmic laser microendoscope ciliary process ablation in the management of neovascular glaucoma. Ophthalmology 1992; 99: $1823-1828$

[23] Murthy G], Murthy PR, Murthy KR et al. A study of the efficacy of endoscopic cyclophotocoagulation for the treatment of refractory glaucomas. Indian J Ophthalmol 2009; 57: 127

[24] Chen J, Cohn RA, Lin SC et al. Endoscopic photocoagulation of the ciliary body for treatment of refractory glaucomas. Am J Ophthalmol 1997; 124: $787-796$

[25] Lima FE, Magacho L, Carvalho DM et al. A prospective, comparative study between endoscopic cyclophotocoagulation and the Ahmed drainage implant in refractory glaucoma. J Glaucoma 2004; 13: 233-237

[26] Francis BA, Berke SJ, Dustin L et al. Endoscopic cyclophotocoagulation combined with phacoemulsification versus phacoemulsification alone in medically controlled glaucoma. J Cataract Refract Surg 2014; 40: 1313-1321

[27] Plager DA, Neely DE. Intermediate-term results of endoscopic diode laser cyclophotocoagulation for pediatric glaucoma. J AAPOS 1999; 3: 131-137

[28] Kraus CL, Tychsen L, Lueder GT et al. Comparison of the effectiveness and safety of transscleral cyclophotocoagulation and endoscopic cyclophotocoagulation in pediatric glaucoma. J Pediatr Ophthalmol Strabismus 2014; 51: 120-127

[29] Prost M. Cyclocryotherapy for glaucoma. Evaluation of techniques. Surv Ophthalmol 1983; 28: 93-100

[30] Meyer J, Soriano J, Janknecht P et al. [Results of cyclocryocoagulation]. Klin Monatsbl Augenheilkd 1994; 205: 14-18

[31] Caprioli ], Strang SL, Spaeth GL et al. Cyclocryotherapy in the treatment of advanced glaucoma. Ophthalmology 1985; 92: 947-954

[32] Brihaye M, Oosterhuis J. Experimental cryoapplication with variations in the pressure exerted on the sclera. Ophthalmic Res 1972; 3: 129-144

[33] Brindley G, Shields M. Value and limitations of cyclocryotherapy. Graefes Arch Clin Exp Ophthalmol 1986; 224: 545-548

[34] Goldenberg-Cohen N, Bahar I, Ostashinski M et al. Cyclocryotherapy versus transscleral diode laser cyclophotocoagulation for uncontrolled intraocular pressure. Ophthalmic Surg 2005; 36: 272-279

[35] Benson M, Nelson M. Cyclocryotherapy: a review of cases over a 10-year period. $\mathrm{Br}$ J Ophthalmol 1990; 74: 103-105

[36] Schlote T, Derse M, Rassmann K et al. Efficacy and safety of contact transscleral diode laser cyclophotocoagulation for advanced glaucoma. J Glaucoma 2001; 10: 294-301

[37] Egbert PR, Fiadoyor S, Budenz DL et al. Diode laser transscleral cyclophotocoagulation as a primary surgical treatment for primary open-angle glaucoma. Arch Ophthalmol 2001; 119: 345-350

[38] Kosoko O, Gaasterland DE, Pollack IP et al. Long-term outcome of initial ciliary ablation with contact diode laser transscleral cyclophotocoagulation for severe glaucoma. The Diode Laser Ciliary Ablation Study Group. Ophthalmology 1996; 103: 1294-1302

[39] Winkler NF, Funk J. [Transscleral cyclophotocoagulation as primary surgical intervention in glaucoma]. Klin Monatsbl Augenheilkd 2013; 230: 353-357

[40] Grueb M, Rohrbach JM, Bartz-Schmidt KU et al. Transscleral diode laser cyclophotocoagulation as primary and secondary surgical treatment in primary open-angle and pseudoexfoliatve glaucoma. Long-term clinical outcomes. Graefes Arch Clin Exp Ophthalmol 2006; 244: 1293-1299 